[Communication]

\title{
准二维无定形碳结构的制备和结构表征
}

\author{
申 珂 ${ }^{1,2}$ 陈 清 ${ }^{1, *}$ 左建民 ${ }^{2, *}$
}

('纳米器件物理与化学教育部重点实验室, 北京大学信息科学技术学院电子学系, 北京 100871;

2美国伊利诺伊大学香槟分校, 材料科学与工程系, 伊利诺伊 61801, 美国)

\begin{abstract}
摘要: 从单层和少层的石墨烯出发, 利用透射电子显微镜中的高能电子束辐照, 可控地制备了准二维完全无 定形和半无定形碳结构. 用高分辨成像和相干的纳米区域电子衍射技术表征了样品结构在高能电子束辐照前 后的变化. 基于实验记录的电子衍射花样得到了样品的原子对分布函数. 分析表明, 在所制备的准二维无定形 结构中, 六元环不再是碳原子的主要排列方式, 碳原子的各阶最近邻间距相对于完美石墨烯中的值有所偏离并 趋向无序; 同时还发现, 锯齿型的碳链结构不易被破坏, 并使得准二维无定形碳结构中还保留了短程有序和可 达 $0.5 \mathrm{~nm}$ 的中程有序.
\end{abstract}

关键词：石墨烯；准二维无定形结构；电子束辐照；高分辨成像；电子衍射；原子对分布函数 中图分类号: $0641 ; 0766$

\section{Fabrication and Structure Characterization of Quasi-2-Dimensional Amorphous Carbon Structures}

\author{
RAN Ke ${ }^{1,2} \quad$ CHEN Qing ${ }^{1, *} \quad$ ZUO Jian-Min ${ }^{2, *}$ \\ ('Key Laboratory for Physics and Chemistry of Nanodevices, Department of Electronics, School of Electronics Engineering and \\ Computer Science, Peking University, Beijing 100871; ${ }^{2}$ Department of Material Science and Engineering, \\ University of Illinois at Urbana-Champaign, Illinois 61801, USA)
}

\begin{abstract}
Quasi-2-dimensional total amorphous and half-amorphous carbon structures are fabricated from single-layer or few-layer of graphene via high-energy electron beam irradiation. Sample structures before and after electron beam irradiation are recorded by high resolution imaging and coherent nano-area electron diffraction using transmission electron microscopy (TEM). The atomic pair distribution functions of the sample are obtained from electron diffraction patterns. In the quasi-2-dimensional amorphous carbon material, the carbon hexagonal ring structure is not the main structural feature anymore, and the low order nearest distances between carbon atoms are slightly different from that in perfect graphene. Although the sample shows a long-range disordered atomic arrangement, it still holds short-range order and even middle-range order extending to $0.5 \mathrm{~nm}$, as many zigzag carbon chains remain after electron beam irradiation.
\end{abstract}

Key Words: Graphene; Quasi-2 dimensional amorphous structure; Electron beam irradiation; High resolution imaging; Electron diffraction; Atomic pair distribution function

Received: April 17, 2012; Revised: May 8, 2012; Published on Web: May 9, 2012.

"Corresponding authors. CHEN Qing, Email: qingchen@pku.edu.cn; Tel: +86-10-62757555. ZUO Jian-Min, Email: jianzuo@illinois.edu; Tel: +1-217-2446504

The project was supported by the National Natural Science Foundation of China (60925003), Ministry of Science and Technology, China (2012CB932702), and Department of Energy, USA (DEFG02-01ER45923).

The experiment was carried out at the Frederick Seitz Materials Research Laboratory Central Facilities, University of Illinois, USA.

国家自然科学基金(60925003), 科技部重大研究计划(2012CB932702)及美国能源部(DEFG02-01ER45923)资助项目

本工作的实验在美国伊利诺伊大学Frederick Seitz材料研究实验室完成.

(C) Editorial office of Acta Physico-Chimica Sinica 


\section{1 引言}

Zachariasen $^{1}$ 最早从理论上提出了无定形结构 的模型. 然而一直以来, 二维的无定形结构被认为 无法稳定存在, 也没有在实验中被实现过. 石墨烯 晶体在实验上的实现, ${ }^{2}$ 不仅打破了 Mermin-Wagner

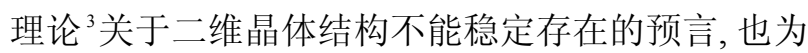
二维无定形结构的实现提供了希望. 与碳纳米管类 似, 石墨烯具有非常优异的电学和机械性能. 随着 合成方法的不断进步, 45 石墨烯在纳米科技等领域 被广泛研究并显示出优越的应用前景, 已经成为纳 米科技领域最具潜力的材料之一. 同时, 研究也表 明, 石墨烯结构中往往存在缺陷, ${ }^{6-8}$ 而这些缺陷会在 很大程度上影响石墨烯的性能. ${ }^{9,10}$ 如果能在石墨烯 结构中有目的地引入缺陷, ${ }^{11,12}$ 则可望实现对其性能 的一种调制.

透射电子显微镜(TEM) 是表征纳米材料的最强 大手段之一. 此外, 在和样品相互作用的过程中, 入 射电子束还可以激发样品中的物理、化学变化, 引 入缺陷以及改变样品结构等等. ${ }^{13,14}$ 研究表明, 高能 电子束辐照可在石墨烯结构中引入不同类型的缺 陷, ${ }^{15}$ 而低电压下的结构表征也可在不破坏样品结 构的前提下获得足够高的分辨率. ${ }^{16}$ 本文将从单层 和少层的石墨烯出发, 结合高分辨成像和相干的纳 米区域电子衍射, 分别利用 200 和 $80 \mathrm{keV}$ 的入射电 子来实现准二维无定形碳结构的产生和表征. 由样 品的电子衍射花样获得的原子对分布函数则可提 供碳原子在其中排列的信息.

\section{2 实验部分}

\section{1 单层和少层石墨烯的制备}

机械剥离是制备单层石墨烯的常用手段之一, ${ }^{17}$
而“Sticky Tape” 是其中最著名的一种. 但是, 靠这种 方法制备的样品很容易被胶带或其上的附着物污 染, 不太适用于高分辨透射电镜研究. 本研究选用 了“Pencil Drawing”的方法: 首先, 利用高定向热解 石墨(HOPG)在硅片上轻轻地划不重复的线; 然后, 将硅片中被 HOPG 划过的表面朝上, 放入装有少量 $N, N$-二甲基甲酰胺(DMF)溶液, 容量为 $600 \mathrm{~mL}$ 的烧 杯中, 并进行小功率超声; 最后, 将溶于 DMF 中的石 墨烯样品转移到透射电镜微栅上. “Pencil Drawing” 方法虽然无法制备大面积的单层石墨烯, 但其制得 的样品比较清洁, 得到的尺度在几十个纳米的单层 或少层石墨烯已经能够满足本研究的需要. 此外, 这种方法还具有方便和经济的优点.

图 1 为利用 “Pencil Drawing”方法制得的石墨 烯样品在不同放大倍数下的透射电镜照片. 可以看 到, 制备的样品中存在足够多的尺寸超过 $10 \mathrm{~nm}$, 具 有完整晶格结构的区域.

\section{2 准二维无定形碳结构的制备和结构表征}

实验中, 准二维无定形材料的制备和结构表征 都是在透射电镜中完成的. 研究表明, 利用 $80 \mathrm{kV}$ 的 入射电压, 既可对石墨烯进行高分辨成像, ${ }^{18}$ 又能有 效地避免样品的辐照损伤. ${ }^{19}$ 因此, 本研究中对样品 结构的记录都选择在 $80 \mathrm{kV}$ 的入射电压下完成. 此 外, 据文献报导, 在石墨烯结构中造成撞击损伤的 入射电子阈值电压约为 100-140 kV. ${ }^{20}$ 因此, 为了在 石墨烯结构中有效地引入缺陷, 实验采用 $200 \mathrm{keV}$ 的入射电子对其进行辐照.

此部分实验的主要思路为: 从单层和少层的石 墨烯出发, 利用高能电子束 $(200 \mathrm{keV})$ 辐照在样品中 引入缺陷, 然后在较低的加速电压下 $(80 \mathrm{kV})$ 结合透 射电镜高分辨成像和相干的纳米区域电子衍射 ${ }^{21}$ 记
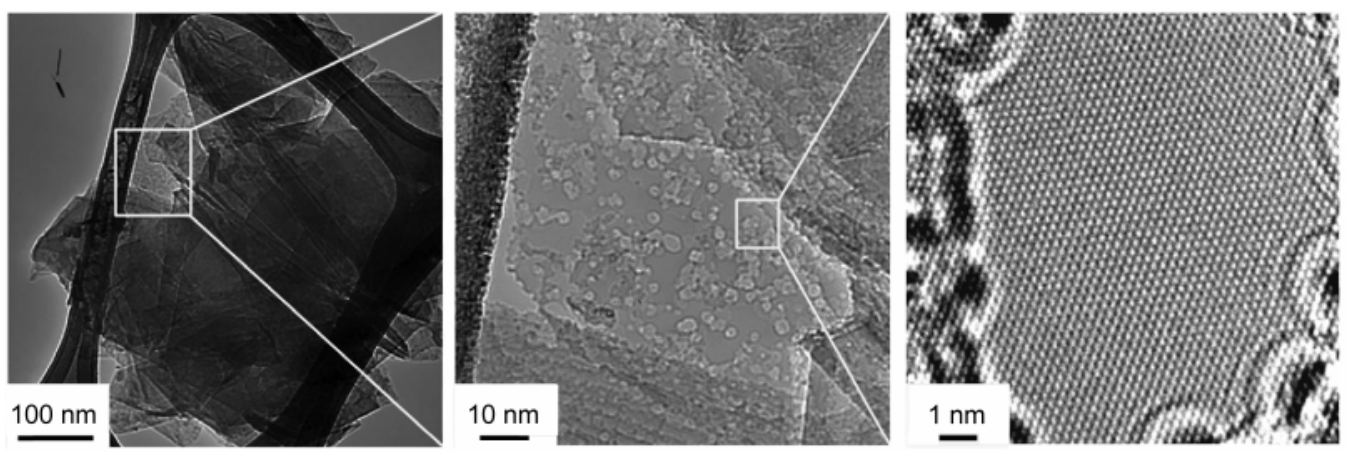

图 1 利用“Pencil Drawing”方法制备的石墨烯样品在不同放大倍数下的透射电镜(TEM)照片

Fig.1 Transmission electron microscopy (TEM) images of the graphene sample prepared by "Pencil Drawing” method at different magnifications 
录样品的结构信息. 所有实验都是在日本电子 JEOL 2200FS 型号的透射电镜上完成的.22

图 $2 \mathrm{a}$ 和 $2 \mathrm{c}$ 为同一片石墨烯样品在经历高能电 子束辐照前后的透射电镜照片. 图 $2 b$ 和 $2 \mathrm{~d}$ 分别为 其相应的电子衍射花样. 可以看到, 经过电子束辐 照, 石墨烯样品中的晶格结构几乎被完全破坏, 但 碳原子的排列还保持了一定的有序性. 通过控制电 子束辐照的条件(如辐照时间和电子束流密度), 实 验还制备了完全无定形的准二维碳结构, 图 2e 为其 相应的电子衍射花样. 很明显, 该样品已经完全失 去了石墨烯的晶格结构.

\section{3 结果和讨论}

由于无定形结构的原子排列不具备长程有序 性, 所以无法像对晶体那样用晶格常数和晶体平移
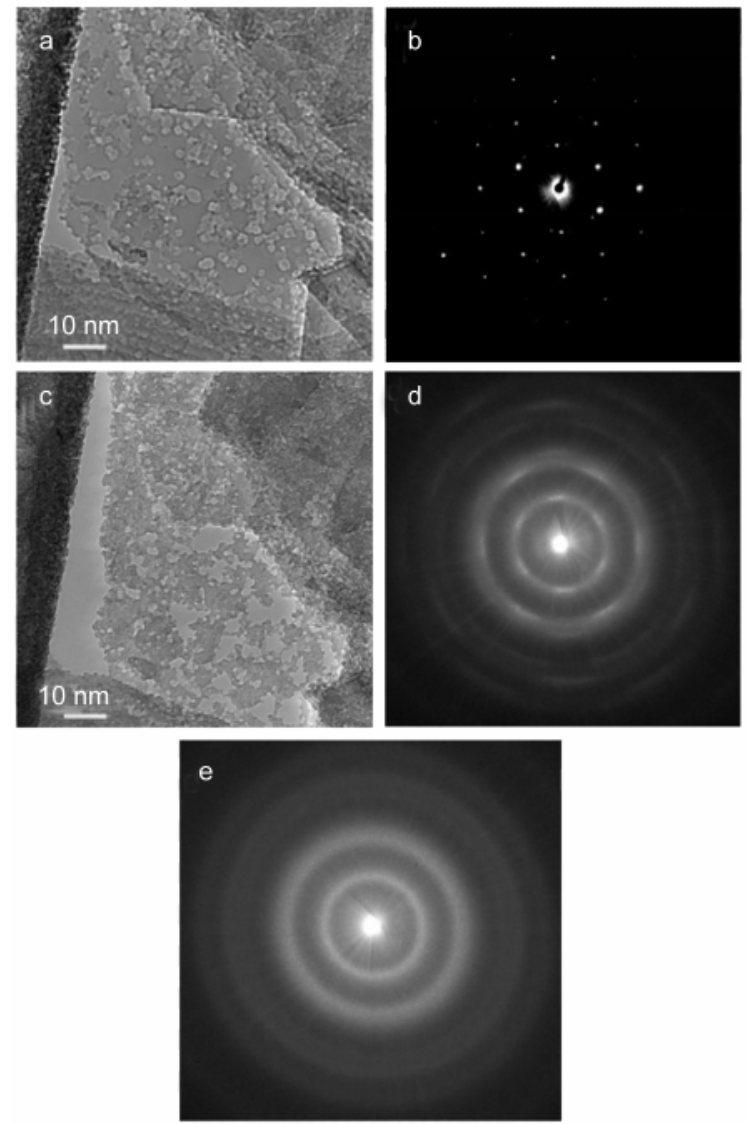

图 2 同一石墨烯样品在电子束辐照前 $(a, b)$ 和后 $(c, d)$ 的 结构 $(a, c)$ 及对应的电子衍射花样 $(b, d)$; 准二维完全 无定形碳结构的电子衍射花样(e)

Fig.2 Recorded structures (a, c) and corresponding electron diffraction patterns $(b, d)$ from a same graphene sample before $(a, b)$ and after $(c, d)$ electron beam irradiation; electron diffraction pattern from a total amorphous quasi-2 dimensional carbon structure (e)
对称性来描述. 因此, 采用原子对分布函数 ${ }^{23}$ 来分析 实验中制备的纳米尺度的准二维无定形结构. 在无 定形材料中, 原子间的相对位置可以由一系列的原 子间距 $\left\{r_{v v}\right\}$ 来描述, 其中 $v$ 和 $v$ 分别代表两个独立的 原子. 在各向同性的系统中, 原子间距的分布可表 示为 $\rho(r)=\rho_{0} g(r)=\sum_{v} \sum_{v} \delta\left(r-r_{v v}\right) / 4 \pi N r^{2}$. 其中, $r$ 是原子间 距, $\rho_{0}$ 为整个系统 (共包含 $N$ 个原子) 的原子数密度, $\rho(r)$ 为原子对密度函数, $g(r)$ 为原子对分布函数. ${ }^{24}$ $J(r) \equiv 4 \pi r^{2} \rho_{0} g(r)$ 被称为径向分布函数. 正是 $g(r)$ 和 $J(r)$ 中包含了丰富的样品结构信息. 例如, $g(r)-r$ 曲线上 峰值出现的位置对应了样品中近邻原子间距的信 息, ${ }^{25}$ 对峰强度的积分可得到样品中原子配位数的 信息, ${ }^{26}$ 而峰的宽度还可提供样品中原子热振动的 信息. ${ }^{27,28}$

样品的原子对分布函数可以从其电子衍射花 样中获得. 首先, 对实验上直接得到的样品衍射花 样进行一系列处理, 包括校准相机常数和图像畸 变, 沿圆周积分, 去除背底噪音以及对信号进行归 一化等, 29,30 可得到样品在倒空间中的结构方程 $S(q)$, 其中 $q$ 为倒空间中的矢量长度. $S(q)$ 和样品在实空间 中的原子对分布函数 $g(r)$ 通过方程 $S(q)=1+$ $\int_{0}^{\infty} G(r) \sin (q r) \mathrm{d} r / q$ 联系起来. 其中, $G(r)=4 \pi \rho_{0} r[g(r)-1]$, 称为约化的原子对分布函数. ${ }^{24}$ 在使用如图 2 显示的 衍射花样时, 我们假设了二维投影近似, 忽略了在 平面外方向上可能的小的原子位移.

图 3 为从如图 2e 所示的电子衍射花样得到的 $G(r)$. 可以看到, 随着 $r$ 的增大, $G(r)$ 曲线在 $r=0.149$, $0.253,0.374,0.491 \mathrm{~nm}$ 处有明显的峰(如图中上方线 段标记), 表明样品中原子的排列存在一定的有序 度, 峰的位置对应了样品中碳原子的各阶最近邻间 距. 而 $G(r)$ 曲线在 $r=0.491 \mathrm{~nm}$ 左右的峰表明, 在衍射 花样中显示为完全无定形的准二维碳结构, 其原子 排列不仅具有短程有序, 还具有到 $0.5 \mathrm{~nm}$ 左右的中 程有序. 如图 3 中插图所示, 在完美石墨烯晶体中, 碳原子前六阶最近邻间距分别为: $r_{1}=0.142 \mathrm{~nm}, r_{2}=$ $0.246 \mathrm{~nm}, r_{3}=0.284 \mathrm{~nm}, r_{4}=0.355 \mathrm{~nm}, r_{5}=0.424 \mathrm{~nm}, r_{6}=$ $0.492 \mathrm{~nm}$. 对比可知, 图 3 中 $G(r)$ 曲线上的峰分别对 应 $r_{1} 、 r_{2} 、 r_{4}$ 和 $r_{6}$. 石墨烯中碳原子的第三近邻, $r_{3}=$ $0.284 \mathrm{~nm}$, 对应碳六元环的直径; 第五近邻, $r_{5}=$ $0.424 \mathrm{~nm}$, 对应单个碳一碳键长加上一个碳六元环 的直径. ${ }^{31}$ 在图 3 中 (如图中箭头所示), $G(r)$ 曲线在 $r_{3}=0.284 \mathrm{~nm}$ 附近几乎为平滑的曲线, 在 $r_{5}=0.424 \mathrm{~nm}$ 


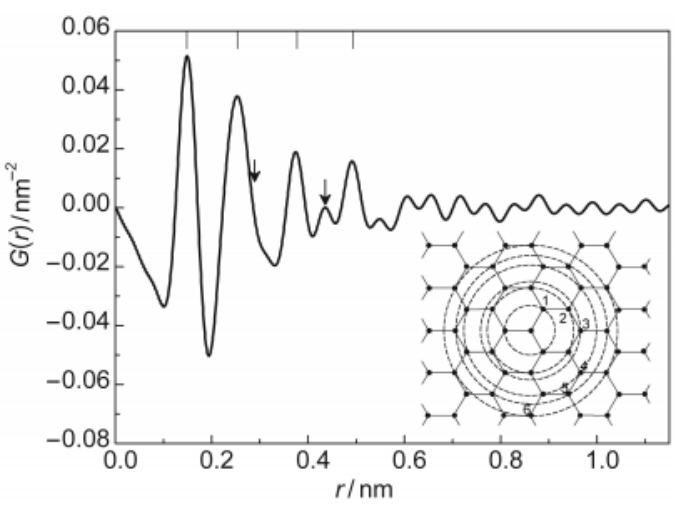

图 3 由图 2e 所示的电子衍射花样得到的样品 约化的原子对分布函数

Fig.3 Reduced atomic pair distribution function obtained from the diffraction pattern shown in Fig.2e

Inset shows the neighborhood of a carbon atom in a perfect graphene.

附近仅有一个假峰(峰值小于 0). 这说明, 在经过电 子束辐照之后, 六元环已经不再是样品中碳原子的 主要排列方式, 取而代之的可能是大量的多元环结 构. 还可以看到, $r_{2} 、 r_{4}$ 和 $r_{6}$ 都与碳原子链的长度相对 应, 说明碳原子链不易被完全破坏, 准二维碳结构 中还存在很多甚至包含 5 个原子的有序碳原子链. 另一方面, 图 3 中 $G(r)$ 曲线给出的各阶碳原子最近 邻间距与完美石墨烯晶体中的数值有所偏离, 这可 能是高能电子束辐照过程中, 样品中的碳一碳键扭 曲或伸长, ${ }^{32}$ 碳原子链发生形变造成的. 随着 $r$ 继续 增大, $G(r)$ 曲线很快衰减为 0 附近的无规则振荡, 这 种迅速的衰减反映了样品中碳原子的排列不再具 有长程有序性。

文献 ${ }^{31}$ 中计算了单层石墨烯晶体的原子对分布 函数. 将 $G(r)$ 曲线中出现的对应于石墨烯中碳原子 的第一、二、四和六阶最近邻间距的峰的峰强和半 高宽分别表示为 $I_{1} 、 I_{2} 、 I_{4} 、 I_{6}$ 和 $\Delta_{1} 、 \Delta_{2} 、 \Delta_{4} 、 \Delta_{6}$, 表 1 比较 了来自完美石墨烯晶体和来自图 3 所示的 $G(r)$ 曲线 上各峰的相对强度和半高宽. 可以看到, 准二维无 定形碳结构的 $G(r)$ 曲线中的各个峰相对第一近邻峰 的相对强度均有明显的降低. 这表明, 在实验样品 中, 碳原子的排列不仅失去了长程有序性, 而且在

表 1 石墨烯晶体与图 3 的实验样品的 $G(r)$ 曲线比较 Table 1 Comparison between the $G(r)$ from graphene and shown in Fig.3

\begin{tabular}{lccccccc}
\hline & $I_{2} / I_{1}$ & $I_{4} / I_{1}$ & $I_{6} / I_{1}$ & $\Delta_{1} / \mathrm{nm}$ & $\Delta_{2} / \mathrm{nm}$ & $\Delta_{4} / \mathrm{nm}$ & $\Delta_{6} / \mathrm{nm}$ \\
\hline graphene & 1.19 & 0.62 & 0.42 & 0.017 & 0.016 & 0.016 & 0.008 \\
amorphous sample & 0.74 & 0.37 & 0.31 & 0.030 & 0.041 & 0.025 & 0.068 \\
\hline
\end{tabular}

$I_{n}$ and $\Delta_{n}$ are the intensity and full width at half maximum of the peak in $G(r)$ corresponding to the carbon atom's $n$th neighboring distance.
中短程范围内, 其有序程度也大大低于石墨烯晶 体. 同时, 样品的 $G(r)$ 曲线中各个峰相对石墨烯晶体 的峰还有明显的展宽, 反映了各特定近邻的碳一碳 原子间距分布范围变大, 即整体趋向无序.

\section{4 结 论}

设计了 $80 \mathrm{kV} \rightarrow 200 \mathrm{kV} \rightarrow 80 \mathrm{kV}$ 的透射电镜工 作流程, 从单层和少层的石墨烯出发, 实现了纳米 尺度的准二维无定形碳结构的可控制备和结构表 征. 基于实验中记录的电子衍射花样得到了准二维 无定形碳结构的原子对分布函数. 原子对分布函数 的分析表明, 高能电子束辐照破坏了石墨烯的晶格 结构, 使得六元环不再是碳原子的主要排列方式. 各阶碳原子最近邻间距相对完整石墨烯的值有所 偏离并趋向无序; 同时还发现, 准二维无定形碳结 构中还存在短程有序和可达 $0.5 \mathrm{~nm}$ 的中程有序.

\section{References}

(1) Zachariasen, W. H. J. Am. Chem. Soc. 1932, 54, 3841. doi: 10.1021/ja01349a006

(2) Novoselov, K. S.; Geim, A. K.; Morozov, S. V.; Jiang, D.; Zhang, Y.; Dubonos, S. V.; Grigorieva, I. V.; Firsov, A. A. Science 2004, 306, 666. doi: 10.1126/science.1102896

(3) Mermin, N. D. Phys. Rev. 1968, 176, 250. doi: 10.1103/ PhysRev.176.250

(4) Li, X. S.; Cai, W. W.; An, J. H.; Kim, S. Y.; Nah, J. H.; Yang, D X.; Piner, R.; Velamakanni, A.; Jung, I.; Tutuc, E.; Banerjee, S. K.; Colombo, L.; Ruoff, R. S. Science 2009, 324, 1312. doi: 10.1126/science. 1171245

(5) Hernandez, Y.; Nicolosi, V.; Lotya, M.; Blighe, F. M.; Sun, Z. Y.; De, S.; Mcgovern, I. T.; Holland, B.; Byrne, M.; Gunko, Y. K.; Boland, J. J.; Niraj, P.; Duesberg, G.; Krishnamurthy, S.; Goodhue, R.; Hutchison, J.; Scardaci, V.; Ferrari, A. C.; Coleman, J. N. Nat. Nanotechnol. 2008, 3, 563.

(6) Banhart, F.; Kotakoski, J.; Krasheninnikov, A. V. ACS Nano 2011, 5, 26. doi: $10.1021 / \mathrm{nn} 102598 \mathrm{~m}$

(7) Hashimoto, A.; Suenaga, K.; Gloter, A.; Urita, K.; Iijima, S. Nature 2004, 430, 870. doi: 10.1038/nature02817

(8) Girit, C. O.; Meyer, J. C.; Erni, R.; Rossell, M. D.; Kisielowski, C.; Yang, L.; Park, C. H.; Crommie, M. F.; Cohen, M. L.; Louie, S. G.; Zettl, A. Science 2009, 323, 1705. doi: 10.1126/ science. 1166999

(9) Huang, P. Y.; Ruiz-Vargas, C. S.; van der Zande, A. M.; Whitney, W. S.; Levendorf, M. P.; Kevek, J. W.; Garg, S.; Alden, J. S.; Hustedt, C. J.; Zhu, Y.; Park, J.; McEuen, P. L.; Muller, D. A. Nature 2011, 469, 389. doi: 10.1038/nature09718

(10) Lahiri, J.; Lin, Y.; Bozkurt, P.; Oleynik, I. I.; Batzill, M. Nat. Nanotechnol. 2010, 5, 326. doi: 10.1038/nnano.2010.53 
(11) Ying, H.; Wang, Z. Y.; Guo, Z. D.; Shi, Z. J.; Yang, S. F. Acta Phys. -Chim. Sin. 2011, 27, 1482. [应红, 王志永, 郭政铎, 施祖进, 杨上峰. 物理化学学报, 2011, 27, 1482.] doi: 10.3866/ PKU.WHXB20110630

(12) Gomez-Navarro, C.; Meyer, J. C.; Sundaram, R. S.; Chuvilin, A.; Kurasch, S.; Burghard, M.; Kern, K.; Kaiser, U. Nano Lett. 2010, 10, 1144. 10.1021/n19031617

(13) Chuvilin, A.; Kaiser, U.; Bichoutskaia, E.; Besley, N. A.; Khlobystov, A. N. Nat. Chem. 2010, 2, 450. doi: 10.1038/ nchem. 644

(14) Ran, K.; Zuo, J. M.; Chen, Q.; Shi, Z. J. ACS Nano 2011, 4, 3367.

(15) Kotakoski, J.; Krasheninnikov, A. V.; Kaiser, U.; Meyer, J. C. Phys. Rev. Lett. 2011, 106, 105505. doi: 10.1103/ PhysRevLett.106.105505

(16) Warner, J. H.; Rummeli, M. H.; Ge, L.; Gemming, T.; Montanari, B.; Harrison, N. M.; Buchner, B.; Briggs, G. A. D. Nat. Nanotechnol. 2009, 4, 500. doi: 10.1038/nnano.2009.194

(17) Novoselov, K. S.; Jiang, D.; Schedin, F.; Booth, T. J.; Khotkevich, V. V.; Morozov, S. V.; Geim, A. K. Proc. Natl. Acad. Sci. U. S. A. 2005, 102, 10451. doi: 10.1073/ pnas. 0502848102

(18) Meyer, J. C.; Kisielowski, C.; Erni, R.; Rossell, M. D.; Crommie, M. F.; Zettl, A. Nano Lett. 2008, 8, 3582. doi: $10.1021 / \mathrm{nl} 801386 \mathrm{~m}$

(19) Egerton, R. F.; Li, P.; Malac, M. Micron 2004, 35, 399. doi: 10.1016/j.micron.2004.02.003

(20) Krasheninnikov, A. V.; Banhart, F. Nat. Mater. 2007, 6, 723. doi: $10.1038 /$ nmat1996
(21) Zuo, J. M.; Gao, M.; Tao, J.; Li, B. Q.; Twesten, R.; Petrov, I. Microsc. Res. Techniq. 2004, 64, 347. doi: 10.1002/jemt.20096

(22) Wen, J. G.; Mabon, J.; Lei, C. H.; Burdin, S.; Sammann, E.; Petrov, I.; Shah, A. B.; Chobpattana, V.; Zhang, J.; Ran, K.; Zuo, J. M.; Mishina, S.; Aoki, T. Microsc. Microanal. 2010, 16 , 183. doi: $10.1017 / \mathrm{S} 1431927610000085$

(23) Cockayne, D. J. H. Annu. Rev. Mater. Sci. 2007, 37, 159. doi: 10.1146/annurev.matsci.35.082803.103337

(24) Egami, T.; Billinge, J. L. Underneath the Bragg Peaks: Structural Analysis of Complex Materials; Pergamon: Amsterdam, 2003; pp 55-99.

(25) Petkov, V.; Jeong, I. K.; Chung, J. S.; Thorpe, M. F.; Kycia, S.; Billinge, S. J. L. Phys. Rev. Lett. 1999, 83, 4089. doi: 10.1103/ PhysRevLett.83.4089

(26) Petkov, V.; Difrancesco, R. G.; Billinge, S. J. L.; Acharya, M.; Foley, H. C. Philos. Mag. B 1999, 79, 1519.

(27) Billinge, S. J. L.; DiFrancesco, R. G.; Kwei, G. H.; Neumeier, J. J.; Thompson, J. D. Phys. Rev. Lett. 1996, 77, 715. doi: 10.1103/ PhysRevLett.77.715

(28) Jeong, I. K.; Proffen, T.; Mohiuddin-Jacobs, F.; Billinge, S. J. L. J. Phys. Chem. A 1999, 103, 921. doi: 10.1021/jp9836978

(29) Ruan, C. Y.; Murooka, Y.; Raman, R. K.; Murdick, R. A.; Worhatch, R. J.; Pell, A. Microsc. Microanal. 2009, 15, 323. doi: $10.1017 /$ S1431927609090709

(30) Chen, H.; Zuo, J. M. Acta Materialia 2007, 5, 1617

(31) McKenzie, D. R.; Green, D. C.; Swift, P. D. Thin Solid Films 1990, 193, 418. doi: 10.1016/S0040-6090(05)80052-5

(32) Li, L.; Reich, S.; Robertson, J. Phys. Rev. B 2005, 72, 184109. doi: 10.1103/PhysRevB.72.184109 